\title{
LA INTENSIDAD DE LA EXTERNALIZACIÓN HOTELERA Y SUS BENEFICIOS Y RIESGOS PERCIBIDOS
}

\author{
Tomás F. Espino-Rodríguez. \\ Juan Carlos Ramírez-Fierro \\ Universidad de Las Palmas de Gran Canaria
}

\section{RESUMEN}

Este trabajo se centra en identificar el papel de la estrategia de externalización en los hoteles. Para ello, se analiza los niveles de externalización actual y deseada. El artículo intenta determinar qué ventajas así como los riesgos que perciben los directivos de esta estrategia. Por otra parte se analiza la relación entre el tamaño, categoría y tipo de hotel con la externalización. Los resultados indican que el nivel de externalización es bastante usado. Por otra parte, los beneficios que perciben los directivos son más tácticos que estratégicos. Existe una relación entre la externalización y el tamaño del hotel.

Palabras clave: externalización; beneficios tácticos y estratégicos; riesgos; hoteles.

\section{The intensity of hotel outsourcing and its perceived benefits and risks}

\section{ABSTRACT}

This study focuses on identifying the role of the outsourcing strategy of hotels. To do so, levels of current and desired outsourcing are analyzed. The paper tries to determine what advantages and risks are perceived by managers of this strategy. In addition, the study analyzes the relationship between the size, category, and type of hotel and outsourcing. The results indicate that the level of outsourcing in hotels is quite high. Moreover, the outsourcing benefits perceived by managers are more tactical than strategic. There is a relationship between outsourcing and the size of hotel.

Keywords: outsourcing; tactical and strategic benefits; risks; hotels.

Recibido: 8 de marzo de 2016

Devuelto para su revisión: 5 de septiembre de 2016

Aceptado: 13 de octubre de 2016

Edificio Departamental de Ciencias Económicas y Empresariales. Universidad de Las Palmas de Gran Canaria. 35017 LAS PALMAS DE GRAN CANARIA (España). E-mail: tomasfrancisco.espino@ulpgc.es 


\section{INTRODUCCION}

La globalización y el alto nivel de competitividad empresarial han puesto a muchas empresas en una posición difícil para mantener y desarrollar las múltiples habilidades y conocimientos necesarios para ser productivos y rentables. Las empresas quieren desarrollar nuevos productos y servicios en el menor tiempo posible que satisfagan las necesidades de sus clientes, y esto se consigue a través de la innovación, aumentando todo ello la dificultad de llevar a cabo todo este desarrollo internamente (Chatzoglou y Sarigiannidis, 2009).

La demanda turística ha evolucionado, el consumidor del producto turístico ha transformado sus hábitos y comportamientos, tiene cada vez un mayor nivel cultural y es consciente de sus necesidades, expectativas y derechos. Esta evolución del turista lo ha transformado en un cliente que exige un alto grado de calidad de los servicios y que demanda la experiencia del consumo mucho más que el propio consumo. Por esta razón la industria hotelera, que ha estado tradicionalmente más centrada en la tangibilidad de los productos, debe centrarse en la actualidad en las necesidades y expectativas del cliente y en lo que espera de su experiencia turística. Al mismo tiempo el entorno se ha vuelto más competitivo, los establecimientos hoteleros compiten entre sí, y especialmente intensa se ha vuelto la competencia entre los destinos turísticos nuevos y los consolidados. Las zonas turísticas compiten por adaptarse a las necesidades de los turistas, intentando proporcionar un alto nivel de calidad, lo que les conduce al crecimiento y desarrollo de su territorio (Tardivo et al., 2013; Shani et al., 2014). El dinamismo del mercado y la alta competitividad requieren de estrategias en la gestión hotelera actual que se centren tanto en el éxito presente como en la inversión en las actividades que promuevan una ventaja competitiva para el éxito futuro.

La externalización es una técnica ampliamente recomendada para mejorar la posición competitiva (Maiga y Jacobs, 2004). Como señala Özdoğan (2006), supone una oportunidad para la mejora de la eficiencia en las empresas, y es una forma adecuada de adaptar los límites de la empresa mediante la reestructuración de sus actividades con el objetivo de estimular el crecimiento de su negocio principal (Bustinza et al., 2010). La externalización también puede proporcionar competencias básicas esenciales en actividades tales como la fabricación y comercialización o funciones de apoyo en otras más especializadas como el servicio de alimentos, mantenimiento, sistemas de información y contabilidad (Gilley et al., 2004).

El crecimiento de la externalización ha sido exponencial y muchas empresas la usan hoy en día (Maiga y Jacobs, 2004; Kakabadse y Kakabadse, 2005; Taylor, 2005). En los últimos tiempos, hemos asistido a la sustitución del concepto tradicional de empresa, en el que las diferentes actividades de la cadena de valor se realizan internamente por los conceptos de la "organización de red" o incluso "organización virtual", donde el número de actividades realizadas internamente es cada vez más bajo (Burn y Ash, 2000;Tetteh y Burn, 2001). En la gestión hotelera el uso de la externalización ha sido recurrente durante los últimos años (Donada y Nogatchewsky, 2009). Frente a una competencia más intensa, los hoteles tienen que sobrevivir mediante la mejora de la calidad y la satisfacción del cliente (Lam y Han, 2005). El protagonismo que ha adquirido la externalización en hoteles es indiscutible. A finales de 2014 se establecía que un 30\% de los cerca de 260.000 empleados que trabajan en los hoteles españoles estaban externalizados, las previsiones son de un incremento medio anual de entre el $10 \%$ y el $15 \%$,elevando al $60 \%$ los trabajadores externalizados para el 
año 2020 (Hosteltur, 2015). Por su parte, la literatura académica sobre externalización hotelera, cuyos orígenes de las primeras investigaciones se sitúan en el año 2000, ha crecido de manera notable desde el año 2005 (Gonzalez et al., 2011). La importancia que ha adquirido la estrategia de externalización en la gestión hotelera convierte en necesaria una mayor investigación en esta área. Por ello, en este trabajo se analiza el nivel de externalización actual y deseado de los diferentes departamentos del hotel, así como los principales beneficios y riesgos que supone la externalización para los directivos. También se estudia la relación entre la externalización con la categoría, el tipo de hotel y el tamaño.

\section{LA EXTERNALIZACIÓN EN LAS EMPRESAS HOTELERAS}

La externalización surgió como práctica en los años 50, pero no fue hasta los años 80 cuando fue ampliamente adoptada como estrategia en las empresas, evolucionando los motivos relacionados con la reducción de costes a una naturaleza más cooperativa, en el cual el coste no es el único, incluso es secundario, criterio para tomar la decisión de externalizar. El término externalización es ampliamente utilizado tanto en el ámbito académico como empresarial. Existe un amplio debate en la literatura sobre su definición (Gilley y Rasheed, 2000). Distintos autores han establecido diferentes conceptos de externalización en la literatura. En el cuadro 1, se observan las definiciones más destacadas de las últimas décadas. Algunos de ellos se refieren en el concepto a las actividades o al tipo de actividades que pueden estar en manos de terceros. En esta línea se encuentran Leeman y Reynolds (2012) que definen la externalización como la utilización por una organización de empresas externas para realizar tareas que normalmente se han realizado internamente. Otros autores definen la externalización desde el punto de vista de la importancia de las actividades para la empresa, es decir, aquellas que no son estratégicas (Espino-Rodríguez y Padrón-Robaina, 2006). Chase et al. (2004) definen la externalización como la transferencia de algunas de las actividades internas y de responsabilidades de decisión desde la empresa hacia suministradores externos. Otros autores como Bailey et al. (2002) la consideran como "una entrega de parte o la totalidad de una actividad y los servicios relacionados a una tercera parte para su gestión”.

Podemos seguir esbozando definiciones de externalización, pero no encontraremos en los autores unanimidad en la misma; sin embargo, las definiciones tienen un punto en común y es que la externalización implica la provisión externa de bienes o servicios que previamente eran realizados en el seno de las organizaciones (Gilley y Rasheed, 2000; Campos, 2001; Ecerkale y Kovanci, 2005; Leeman y Reynolds, 2012). Casi todos los autores coinciden también que las empresas deben centrarse en aquellas actividades en las que poseen una ventaja competitiva sostenible, externalizando aquellas otras en las que las compañías de la competencia posean a su vez dicha ventaja competitiva (Gilley y Rasheed, 2000; Lam y Ham, 2005; Espino-Rodríguez y Padrón-Robaina, 2006).

Los hoteles son más competitivos no solamente por la reducción de costes de la externalización, sino también por la influencia de otras prioridades competitivas dentro de la estrategia de operaciones, tales como la calidad, la flexibilidad y un mejor servicio (Espino-Rodríguez y Padrón-Robaina, 2004). Anteriores estudios han demostrado que para que la estrategia de externalización tenga un uso más generalizado debe tener mayor efecto en otras prioridades competitivas diferentes a la reducción de costes (Lam y Han, 2005). 


\section{Cuadro 1 \\ EVOLUCIÓN DEL CONCEPTO DE EXTERNALIZACIÓN}

\begin{tabular}{|c|c|}
\hline Autor & Concepto de externalización \\
\hline $\begin{array}{l}\text { Gilley y Rasheed } \\
(2000)\end{array}$ & $\begin{array}{l}\text { Es la substitución de actividades realizadas internamente por la } \\
\text { adquisición externa de estas, aunque la firma posea las capacidades } \\
\text { financieras y de gestión necesarias para realizarlas internamente. }\end{array}$ \\
\hline Campos (2001) & $\begin{array}{l}\text { Consiste en contratar a un proveedor externo una tarea que } \\
\text { anteriormente se realizaba en la propia organización e incluso de } \\
\text { nuevas actividades. }\end{array}$ \\
\hline Bailey et al. (2002) & $\begin{array}{l}\text { Entrega de parte o la totalidad de una actividad y los servicios } \\
\text { relacionados a una tercera parte para su gestión para obtener el } \\
\text { resultado requerido. }\end{array}$ \\
\hline $\begin{array}{l}\text { Quélin y Duhamel } \\
(2003)\end{array}$ & $\begin{array}{l}\text { La operación de cambio de una transacción previamente gobernada } \\
\text { internamente a un proveedor externo a través de un contrato a largo } \\
\text { plazo, y que implica la transferencia al vendedor. }\end{array}$ \\
\hline $\begin{array}{l}\text { McCarthy y } \\
\text { Anagnostou (2004) }\end{array}$ & $\begin{array}{l}\text { No consiste solamente en la compra de productos o servicios de } \\
\text { fuentes externas sino que transfiere también la responsabilidad de } \\
\text { funciones de negocio y a menudo el conocimiento asociado (tácito } \\
\text { y codificado) a la organización externa. }\end{array}$ \\
\hline Chase et al. (2004) & $\begin{array}{l}\text { Transferencia de algunas de las actividades internas y } \\
\text { de responsabilidades de decisión desde la empresa hacia } \\
\text { suministradores externos. }\end{array}$ \\
\hline Mol et al. (2005) & $\begin{array}{l}\text { La adquisición de suministros de entidades jurídicamente } \\
\text { independientes (proveedores). }\end{array}$ \\
\hline $\begin{array}{l}\text { Ecerkale y Kovanci } \\
(2005)\end{array}$ & $\begin{array}{l}\text { La externalización consiste en que una empresa ofrece un servicio } \\
\text { a través de otra empresa especializada en dicho servicio en vez de } \\
\text { producirlo internamente. }\end{array}$ \\
\hline Lam y Han (2005) & $\begin{array}{l}\text { La externalización es una estrategia en la que una empresa utiliza } \\
\text { un proveedor especializado, estableciendo una relación en forma } \\
\text { de alianza, en la que el proveedor desempeñará ciertas funciones } \\
\text { de la empresa, en un intento de reducir el riesgo y los costes y } \\
\text { mejorar la eficiencia. }\end{array}$ \\
\hline $\begin{array}{l}\text { Espino-Rodríguez } \\
\text { y Padrón-Robaina } \\
(2006)\end{array}$ & $\begin{array}{l}\text { Es una decisión estratégica que supone la contratación externa de } \\
\text { determinadas actividades no estratégicas de la empresa, necesarias } \\
\text { para la fabricación de bienes o prestación de servicios, mediante } \\
\text { el establecimiento de acuerdo o contratos con las empresas más } \\
\text { capacitadas para realizar dichas actividades con el fin de mejorar la } \\
\text { ventaja competitiva. }\end{array}$ \\
\hline $\begin{array}{l}\text { Moschuris y } \\
\text { Kondylis (2006) }\end{array}$ & $\begin{array}{l}\text { La externalización es el proceso de contratación de una compañía } \\
\text { externa para suministrar un servicio previamente realizado por el } \\
\text { personal. }\end{array}$ \\
\hline
\end{tabular}




\begin{tabular}{|l|l|}
\hline \multicolumn{1}{|c|}{ Autor } & \multicolumn{1}{c|}{ Concepto de externalización } \\
\hline Oshri et al.(2011) & $\begin{array}{l}\text { Es la contratación con un proveedor para la gestión y realización } \\
\text { de una cierta cantidad de trabajo, por un período de tiempo } \\
\text { especificado, coste y nivel de servicio. }\end{array}$ \\
\hline $\begin{array}{l}\text { González et al. } \\
(2011)\end{array}$ & $\begin{array}{l}\text { La externalización como una relación contractual entre una empresa } \\
\text { y su proveedor -suministrador de algún servicio- mediante la cual } \\
\text { este proveedor asume el compromiso de prestarle el servicio }\end{array}$ \\
\hline $\begin{array}{l}\text { Leeman y Reynolds } \\
(2012)\end{array}$ & $\begin{array}{l}\text { La externalización es la utilización por una organización de } \\
\text { empresas externas para realizar tareas que normalmente se han } \\
\text { realizado internamente. }\end{array}$ \\
\hline $\begin{array}{l}\text { Dolgui y Proth } \\
(2013)\end{array}$ & $\begin{array}{l}\text { La externalización es definida como el acto de obtener productos } \\
\text { semi-terminados, productos finales o servicios de una compañía } \\
\text { externa si éstos han sido tradicionalmente realizados internamente. }\end{array}$ \\
\hline $\begin{array}{l}\text { Promsivapallop et } \\
\text { al. (2015) }\end{array}$ & $\begin{array}{l}\text { Es la realización por parte de proveedores independientes de la } \\
\text { totalidad o parte de actividades internas de la empresa que podrían } \\
\text { ser, o que previamente han sido, llevadas a cabo por la propia } \\
\text { empresa. }\end{array}$ \\
\hline
\end{tabular}

Fuente: elaboración propia.

La externalización se ha convertido en una estrategia clave en la gestión hotelera durante los últimos años (Donada y Nogatchewsky, 2009), lo cual no es extraño teniendo en cuenta las peculiaridades de este sector (Lamminmaki, 2008). Los hoteles son organizaciones complejas por la diversidad de tareas que tienen lugar (limpieza, reservas, lavandería, animación, cocina, etc.). Esto obliga al directivo a desarrollar muchos roles, funciones y habilidades, haciéndole muy difícil identificar sus competencias básicas o las ventajas competitivas derivadas de ellas (Gonzalez et al., 2011). Por consiguiente la dirección de un hotel se torna compleja, y hay una gran necesidad de los directivos hoteleros de no distraer su atención de las actividades "core" o "básicas" (Domberger, 1998; Lamminmaki, 2008). La externalización supone una opción para minimizar el problema de la complejidad hotelera, ya que muchas de las actividades del hotel pueden ser subcontratadas (Crichton y Edgar, 1995). Esto puede que no ocurra en otros sectores diferentes al hotelero, donde las actividades pueden ser más análogas y más difícil de externalizar al no ser tan diferentes entre sí.

Es necesario definir en primer lugar los límites de la organización para decidir las actividades que puede realizar internamente el hotel y cuales externamente (McIvor, 2005). La importancia de la externalización por motivo de las competencias básicas está ampliamente reconocido en las investigaciones realizadas en el área de la dirección hotelera (Guerrier y Lockwood, 1989; Hottman y Adams, 1996; Johns y Lee-Ross, 1996; Hemmington y King, 2000; Lam y Han, 2005; Espino-Rodríguez y Gil-Padilla, 2005; Espino-Rodríguez y Padrón-Robaina, 2005; Bolat y Yilmaz, 2009; Donada y Nogatchewsky, 2009). Los directivos deberían desarrollar sus competencias básicas como una estrategia de bloqueo de sus competidores y evitar externalizar estas competencias o facilitar el acceso a sus suministradores a los conocimientos y habilidades básicas de 
las mismas (Quinn y Hilmer, 1994). No todas las actividades pueden ser externalizadas ya que puede suponer una pérdida de ventaja competitiva (Bettis et al., 1992). Por ello, es de vital importancia tomar la decisión correcta con un enfoque estratégico a largo plazo (Şahin y Berberoglu, 2011). Se hace necesario por tanto que el directivo hotelero sea capaz de distinguir cuáles de sus actividades son básicas y las que no lo son, para no soportar riesgos innecesarios que puedan influir negativamente en la ventaja competitiva. Que el hotel se centre en sus actividades básicas es uno de los efectos positivos de la externalización ampliamente recogido en la literatura existente (Prahalad y Hamel, 1990; Dess et al., 1995; Arnold, 2000). Estas actividades básicas, en las que el hotel es más eficiente, deben ser el centro de atención de los directivos, ya que su desarrollo interno supone una mayor flexibilidad y rapidez del hotel para adaptarse a los cambios del mercado (Quinn, 1999). Centrarse en este conjunto de operaciones basadas en el conocimiento y habilidades críticas permiten mejorar la calidad y diferenciar su servicio (Espino-Rodríguez et al., 2012). Además la externalización de las actividades no básicas permite incrementar la atención en las actividades estratégicas, lo que puede repercutir en una mayor flexibilidad en las operaciones y la posibilidad de disponer de recursos para otros propósitos (Espino-Rodríguez y Padrón-Robaina, 2004).

En la literatura existen diversos estudios sobre los efectos positivos de la externalización en el sector servicios (Bolat y Yilmaz, 2009; Espino-Rodríguez y Padrón-Robaina, 2005; Yildiz y Hatun, 2014). Esta estrategia ha sido reconocida como una estrategia positiva que conlleva a una reducción de costes (Bengtsson y Dabholkar, 2009; Kroes y Ghosh, 2010), lo que supone también una mayor eficiencia (Özdoğan, 2006). Al mismo tiempo aporta al hotel una capacidad de adaptación a sus necesidades según la demanda, convirtiendo los costes fijos en variables (Espino-Rodríguez et al., 2012). Como señalan Delmotte y Sels (2008), la externalización es también una alternativa para reducir el volumen de personal en un sector caracterizado por un uso de mano de obra intensiva. También puede proporcionar una solución a la volatilidad del mercado por el carácter cíclico y estacional de la industria hotelera (Lamminmaki, 2009).

\section{LOS BENEFICIOS TÁCTICOS Y ESTRATÉGICOS DE LA EXTERNALIZACIÓN}

Los beneficios que justifican el uso de la externalización se resumen en dos grandes grupos: el primero incluye factores relacionados con la reducción de costes y otros aspectos que tienen que ver con el corto plazo, dando lugar a los beneficios tácticos de la externalización, es decir, el enfoque económico de la externalización; y el segundo grupo de beneficios está relacionado con aspectos estratégicos de la empresa relacionados principalmente con la obtención de mejora de sus recursos y capacidades y la mejora de la ventaja competitiva (Quinn y Hilmer, 1994). Como señalan Redondo-Cano y CanetGiner (2010) cada uno de estos enfoques (económico y estratégico), están asociados con diferentes perspectivas teóricas en el estudio de la externalización. Mientras que el enfoque económico está relacionado con la Teoría de los Costes de Transacción (TCE), el enfoque estratégico está vinculado con la visión de la empresa basada en los recursos (RBV). Estos son enfoques complementarios a la hora de explicar el fenómeno de la externalización. 
El análisis de las principales razones que llevan a las empresas a externalizar algún tipo de actividades es una cuestión que ha sido suficientemente analizada en la literatura especializada (Quinn y Hilmer 1994; Klepper y Jones 1997; Poppo y Zenger 1998; Piachaud 2002; Kakabadse y Kakabadse 2005). Esta decisión de externalizar puede ser evaluada una y otra vez mediante multitud de factores (motivos) que pueden ser tanto de carácter estratégicos como tácticos y están en relación con las metas y estrategias de la organización como la reducción de costes, el enfoque en su negocio principal, el aseguramiento de la flexibilidad empresarial, la expansión, y la obtención de una ventaja estratégica para apoyar los objetivos de negocio (Varadarajan, 2009; Kroes y Ghosh, 2010).

Los factores económicos han sido siempre los principales protagonistas en las investigaciones sobre los motivos de la externalización (Holcomb y Hitt, 2007; Lamminmaki, 2011). La externalización mejora los costes de producción por las ventajas en coste o el aprovechamiento de las economías de escala que se pueden obtener por el nivel de producción del proveedor. Asimismo, con la externalización las empresas pueden llegar a tener un menor punto de equilibrio por la reducción de los gastos generales ya que no tienen que invertir en ciertas instalaciones y equipamientos (Farrel, 2005; Bunyaratavej et al., 2007; Lewin et al., 2009). Estos factores económicos o tácticos son en muchos casos los protagonistas de la externalización hotelera, ya que muchas de las razones de la externalización de un hotel están relacionadas con cubrir necesidades urgentes de personal, reducir los costes e inversiones en actividades que no son básicas. Además la externalización permite transformar los costes fijos en variables, especialmente útil en servicios con demanda estacional como el hotelero.

Estos factores económicos también suponen una mejora en la capacidad de respuesta a la variabilidad de la demanda gracias a las economías de escala de los proveedores, al reducir al mínimo las inversiones financieras (Bengtsson y Dabholkar, 2009). Algunas investigaciones empíricas han sugerido también que las empresas que externalizan incluso alcanzan mejoras superiores en costes que las empresas integradas verticalmente (Gilley y Rasheed, 2000; Gilley et al., 2004). Las razones de tipo económico o tipo táctico incluyen el ahorro de costes y la eficiencia o los problemas de capacidad a corto plazo (Redondo-Cano y Canet-Giner, 2010). En nuestro trabajo centramos la percepción de estos beneficios tácticos en que la externalización permite al hotel la reducción de costes, ser más eficiente, cubrir las necesidades urgentes de personal e incrementar la rentabilidad del hotel.

Para externalizar en el mercado actual se hace necesario considerar otros aspectos y no solamente de tipo económicos y operacionales (Hu y Cai, 2004). Aunque el ahorro en costes es el argumento más utilizado en muchos casos de externalización, la búsqueda de flexibilidad, así como la obtención de ventajas estratégicas están adquiriendo una gran relevancia (Redondo-Cano y Canet-Giner, 2010). La decisión de externalizar puede ayudar a la empresa a liberar los recursos necesarios para centrarse en los aspectos más importantes e invertir en nuevos o mayores procesos de retorno o en nuevas oportunidades (Powell et al., 2006). Aspectos como calidad, flexibilidad o la implementación de nuevas tecnologías juegan un papel fundamental para mantener la competitividad de la empresa. Al mismo tiempo es necesario proporcionar servicios de calidad y prestar 
especial atención a las necesidades y satisfacción del cliente (Santoro, 2015). Los beneficios estratégicos de la externalización están en relación con la adquisición de recursos, capacidades y habilidades que proporciona el proveedor, con mayor conocimiento, experiencia y habilidades organizativas en la prestación del servicio. La externalización permite el acceso a empresas especializadas que pueden añadir calidad al producto o servicio, hacerlas más flexibles, reducir su tamaño y los costes burocráticos innecesarios (Redondo-Cano y Canet-Giner, 2010). La externalización influye en la mejora de los conocimientos y la innovación al aprovechar los recursos y capacidades de los suministradores (Tallman y Chacar, 2011). Según Redondo-Cano y Canet-Giner (2010) una consideración estratégica de la externalización considera todas las razones que una organización emplea para apoyar su ventaja competitiva y consolidar su posición en el mercado. En nuestro trabajo se analizan varios beneficios estratégicos de la externalización que abarcan desde la posibilidad de que el hotel se centre en sus actividades básicas hasta el incremento de la calidad de los servicios, pasando por la mejora del desempeño o la posibilidad de que el personal del hotel pueda disponer de más tiempo para dedicarlo a las actividades básicas del establecimiento.

La literatura existente nos muestra que en la mayoría de las decisiones de externalización las razones estratégicas son más relevantes que las tácticas. Sin embargo, como señalan Redondo-Cano y Canet-Giner (2010), algunas actividades combinan ambas razones, como en el caso de las actividades de selección y reclutamiento en la investigación de Ordini y Silvestri (2008) o los servicios hoteleros en el trabajo de EspinoRodríguez y Padrón-Robaina (2005). A pesar del enfoque estratégico que ha adquirido la externalización, los motivos tácticos no pueden ser obviados en la decisión de externalizar, de hecho ambos enfoques son compatibles en muchas actividades (Redondo-Cano y Canet-Giner, 2010). En este sentido, la investigación de Kakabadse y Kakabadse (2005) ya sugería que se debe prestar atención tanto al carácter táctico como estratégico de la externalización para lograr una mayor ventaja competitiva.

\section{LOS RIESGOS ESTRATÉGICOS DE LA EXTERNALIZACIÓN}

La estrategia de externalización puede conllevar determinadas ventajas, pero también puede suponer factores de riesgos que han sido reconocidos en la literatura y que pueden desmotivar su uso (Espino-Rodríguez et al., 2012). Los beneficios de la externalización pueden verse disminuidos fácilmente por varios factores de riesgos cuando se externalizan actividades relacionadas con las tecnologías de la información (Tafti, 2005). Un uso inapropiado de la externalización y un incremento en la misma, ocasionados en muchos casos por una falta de competitividad o deficientes capacidades del suministrador, pueden originar que los hoteles puedan iniciar un proceso de declive que puede dejarlos sin las capacidades y habilidades necesarias para competir y/o ofrecer un servicio de calidad (Espino-Rodríguez et al., 2012). Con el fin de eliminar los posibles efectos negativos de la externalización se debe de tomar en cuenta todas las variables en el proceso de la toma de decisiones de la externalización (Yildiz y Hatun, 2014).

Estos posibles efectos negativos pueden aparecer por ejemplo cuando se han externalizado actividades críticas (Quélin y Duhamel, 2003; Hoecht y Trott, 2006) que suponen 
un gran riesgo para la ventaja competitiva como las que suponen un contacto directo con el cliente o aquellas que pueden derivar en competencias básicas, encontrándonos ante una posible pérdida de destrezas o habilidades críticas para la innovación (McIvor, 2005). A este respecto, Espino-Rodríguez y Gil-Padilla (2007) señalan que la externalización de los sistemas de información en los establecimientos hoteleros les priva de las capacidades y conocimientos necesarios para obtener una ventaja competitiva. Una vez que se limita o cesa la inversión en capacidades críticas puede ser difícil su renovación o la generación de otras (Espino-Rodríguez et al., 2012). Por lo tanto la externalización puede erosionar el potencial de la organización para el aprendizaje, especialmente en aquellas actividades que son necesarias para desarrollar su negocio principal (Lei y Hitt, 1995).

Esto puede suponer un menor rendimiento para el hotel, menor capacidad de innovación y mayores riesgos que podrían influir en la capacidad requerida para identificar y explotar nuevas oportunidades de negocio (Espino-Rodríguez et al., 2012). Sin embargo, este riesgo puede disminuir si por parte de la dirección del hotel se ha realizado un profundo análisis de las actividades y su grado de influencia en la ventaja competitiva. La externalización de actividades críticas puede suponer también la pérdida de cierta flexibilidad para introducir nuevos productos o servicios en el hotel, ya que a menudo se pierde la capacidad de aprender y explotar los cambios tecnológicos, dependiendo el hotel del proveedor totalmente. Esta excesiva dependencia del proveedor puede dificultar la creación del conocimiento y la transferencia del mismo a través de las diferentes funciones o departamentos de la empresa, al verse limitado con la externalización el surgimiento de nuevas e inesperadas soluciones que surgen de las interacciones entre las personas especializadas en diferentes actividades o departamentos (Quinn y Hilmer, 1994). Sin embargo, si el hotel y el proveedor establecen una relación fluida y bidireccional entre los empleados de ambas partes, la base de conocimiento puede ser mucho mayor.

La externalización puede suponer también una pérdida de control y autonomía en el desarrollo del servicio. Puede llegar a ocurrir que el hotel externalice actividades que el proveedor no llegue a suministrar en los términos de cantidad y calidad acordados. Llegado a este caso, y ante la intención de recuperar la actividad internamente, podemos encontrarnos ante la incapacidad de reconstruir la operación subcontratada al haber perdido las habilidades y capacidades necesarias para volver a desarrollarla internamente. Con el fin de evitar futuros problemas con el proveedor, el acuerdo de externalización debe incluir todos los detalles, condiciones y precauciones necesarias que permitan a ambas partes cumplir con lo estipulado en dicho acuerdo (Yildiz y Hatun, 2014). Esta pérdida de control también puede estar relacionada con el bajo número de proveedores existentes.

La difusión de secretos industriales o información confidencial es también un riesgo importante que la empresa adquiere a la hora de decidir externalizar una actividad (Redondo-Cano y Canet-Giner, 2010). El proveedor puede usar la información en contra del hotel, sometiéndole a presión o chantaje para modificar las condiciones establecidas (por ejemplo un incremento de precios), la aparición del oportunismo conduce a un incremento de los costes de transacción (Lamminmaki, 2011). A este respecto, Lammin- 
maki (2007) indica que algunos directivos hoteleros perciben riesgos para externalizar actividades, como las que tienen que ver con la limpieza de zonas comunes y habitaciones debido a la incertidumbre de que estas tareas se realicen adecuadamente.

Toda externalización supone una reestructuración en la empresa que lleva a cabo esta estrategia y puede provocar malestar en la plantilla de trabajadores (Belcourt, 2006). Muchas de las decisiones de externalización pueden ser irreversibles ya que habremos perdido las habilidades y capacidades, sobre todo si prescindimos del personal experimentado. El personal puede ver su puesto de trabajo amenazado; por tanto no es extraña esta posible oposición a la externalización (González et al., 2005; Brooks, 2006). Puede ocurrir también que la situación de incertidumbre que puede generar la externalización conlleve a una bajada de productividad, desmotivación, inseguridad, etc. en el personal del departamento en dónde se va a llevar a cabo la estrategia de externalización (González et al., 2005). En otras ocasiones la externalización puede conllevar una serie de costes ocultos que son aquellos costes que de manera explícita no aparecen en el contrato de externalización pero que se darán inevitablemente a lo largo de la vida del contrato, como por ejemplo: costes de búsqueda del proveedor correcto, costes de transición (el tiempo invertido en traspasar tareas de cliente a proveedor), y los costes de coordinación y control (Barthélemy, 2001).

En algunas ocasiones las decisiones son irreversibles si no se dispone de la infraestructura y los recursos humanos necesarios para desarrollar internamente de nuevo las actividades y servicios externalizados (Barthélemy, 2001). Existen tres razones fundamentales para la irreversibilidad de la decisión de externalizar: el alto coste de recuperar la actividad para desarrollarla internamente, la dificultad para atraer los recursos humanos necesarios y el tiempo requerido en el proceso (Clark et al., 1995).

\section{METODOLOGÍA}

\section{1. Ámbito de la investigación y muestra}

El ámbito de investigación elegido fue el destino turístico de Canarias, que representa uno de los principales destinos de invierno de Europa, y que obtuvo un máximo histórico de casi 13 millones de turistas en el año 2014, lo que representa un aumento de casi ochocientos cincuenta mil turistas con respecto al ejercicio anterior (FRONTUR 2014, Instituto de Estudios Turísticos). Dentro de la Comunidad Autónoma de Canarias se seleccionó la isla de Gran Canaria que ocupa el quinto puesto a nivel nacional con 3.580.000 turistas en 2014 (FRONTUR, 2014), y con 16.442.176 pernoctaciones en plazas hoteleras, una ocupación del 72,73\% y una estancia media de 6,34 días conforme a los datos aportados por el Instituto Nacional de Estadística (INE, 2014). Nuestra investigación se centró en los establecimientos de sol y playa de categorías 3,4 y 5 estrellas de los municipios turísticos de San Bartolomé de Tirajana y Mogán. Cabe destacar que el municipio de San Bartolomé de Tirajana, en el que se encuentra el grueso de la oferta alojativa, es el tercer municipio español por número de pernoctaciones con 11.339.224 (INE, 2014). 
Determinado el ámbito de investigación, procedimos a identificar el número de establecimientos hoteleros de 3 a 5 estrellas de sol y playa existentes. Se elaboró un listado con datos de estos hoteles después de conciliar y actualizar diversas bases de datos (Sistema Informático Turístico TURIDATA de la Consejería de Turismo del Gobierno de Canarias y Patronato de Turismo de Gran Canaria, 2014), obteniéndose un número de hoteles registrados igual a 71 .

Para obtener los datos de la investigación nos dirigimos a los hoteles mediante una encuesta personal en la que los directivos contestaron a diferentes cuestiones planteadas sin intervención directa del investigador, salvo para realizar algún tipo de aclaración o duda suscitada en la realización del mismo. Una vez que el directivo accedía a mantener una entrevista para la realización de la encuesta, le pedíamos una cita (día y hora) para acudir a su lugar de trabajo (el hotel). De los 71 hoteles que configuraban la población de análisis de esta investigación, 63 hoteles participaron en el estudio con 77 encuestas personales realizadas por sus directivos. La unidad de análisis para nuestro estudio es el director y subdirector, pero nos hemos encontrado durante la realización del trabajo de campo con una realidad en la gestión hotelera actual muy distinta a los organigramas y estructuras gerenciales tradicionales. En la actualidad el sector hotelero, bien por eficacia operativa, costes o la situación de crisis vivida en los últimos años, tiende a una estructura más sencilla, con organigramas más planos, es por ello que se obtuvieron 77 encuestas de 63 establecimientos. Nos hemos encontrado con casos muy llamativos durante la realización del trabajo de campo, como el del director que gestionaba 3 hoteles de una misma cadena y que no disponía de la figura de subdirector para ayudarle en su labor, o los de una cadena hotelera que había suprimido la figura de subdirector en los establecimientos de menos de 400 habitaciones, sin olvidarnos de múltiples casos en que los directores eran responsables de dos establecimientos al mismo tiempo, encontrando incluso algún caso en que esos establecimientos se encontraban en localidades distintas (San Bartolomé de Tirajana y Mogán). De los 63 hoteles que participaron, 22 hoteles son de 3 estrellas, 34 hoteles de 4 estrellas y 7 hoteles de 5 estrellas. Del total de hoteles, 53 hoteles analizados son de cadena, donde 26 hoteles son de cadena nacional y 27 de cadena internacional. Con respecto al tamaño de los hoteles analizados destacar que 29 hoteles tienen menos de 250 habitaciones, 26 hoteles disponen entre 250 habitaciones y 500 y 8 hoteles más de 500 habitaciones.

A pesar de estas particularidades en la gestión hotelera actual, obtuvimos una tasa real de respuesta del $88,73 \%$, que incluye la mayoría de los hoteles turísticos de San Bartolomé de Tirajana y Mogán, por lo que la representatividad de la muestra es alta. En las encuestas realizadas se encuentran representadas 18 cadenas hoteleras, la mayoría de carácter internacional, lo que le confiere un valor añadido a los datos obtenidos en nuestra investigación y sugiere que el perfil de los encuestados es el adecuado para la validez de nuestro estudio.

\subsection{Medida de las variables}

Para la elaboración del cuestionario se realizó una revisión de la literatura empírica y teórica acerca de cómo medir el nivel de externalización de las actividades, los dife- 
rentes beneficios tácticos, estratégicos y los riesgos de externalizar. El cuestionario fue pre-testado por investigadores con experiencia en el sector hotelero y directivos, lo que permitió mejorar la claridad y la redacción de los ítems usados.

Grado de externalización de la actividad. Una actividad puede estar total o parcialmente externalizada, dado que puede estar formada por un conjunto de tareas o subactividades (Poppo y Zenger, 1998; Carey et al., 2006; McIvor, 2008). Para cumplir con los objetivos propuestos, identificamos el nivel de externalización actual y deseado de cada una de las 12 actividades usadas en el estudio. Como en estudios previos de externalización (Espino-Rodríguez et al., 2012; Espino-Rodríguez y Chun-Lai, 2014; Wan y Yen-Lun Sun, 2010), se preguntó a los encuestados que indicaran el grado de externalización de una actividad en una escala tipo Likert de 1 a 7 , donde el 1 significa que ninguna parte de la actividad está externalizada y el 7 que la actividad está totalmente externalizada. Para la externalización deseada planteamos una cuestión que indicara el grado de externalización que debería tener esa actividad, también en una escala de 1 a 7 , donde 1 indicaría que no externalizaría la actividad y el 7 que externalizaría la actividad totalmente.

Beneficios tácticos y estratégicos de la externalización. El propósito de este conjunto de preguntas era identificar los principales beneficios que percibían los directivos de la estrategia de externalización de actividades. La revisión de la literatura teórica y empírica sobre los principales beneficios estratégicos y tácticos que permite la externalización (Ford y Farmer, 1986; Saunder et al., 1997; Espino-Rodríguez y Padrón Robaina, 2005; Bustinza et al., 2010; Espino-Rodríguez et al., 2012) nos ha permitido crear una escala multidimensional que incorpora ítems relacionados con la reducción de los costes, la flexibilidad, el acceso a recursos y capacidades y el enfoque en las competencias básicas entre otros. A este respecto, pedíamos a los encuestados que expresaran el grado de acuerdo o desacuerdo en una escala numérica tipo Likert de 1 a 7 con 4 afirmaciones para los beneficios tácticos y 8 afirmaciones para los beneficios estratégicos.

Riesgos estratégicos. Para medir los riesgos estratégicos que supone externalizar actividades se añadió una pregunta en el cuestionario. Para ello, y después de la revisión de la literatura teórica y empírica relacionada con los principales riesgos de la externalización como son la pérdida del control de actividades y de su calidad, así como la pérdida de recursos y capacidades (Venkatesan, 1992; Lei y Hitt, 1995; Quinn y Hilmer, 1994; Espino-Rodríguez y Padrón-Robaina, 2005; Espino-Rodríguez et al., 2012), presentamos a los encuestados un conjunto de 10 afirmaciones en una escala subjetiva numérica tipo Likert de 7 puntos en el que el directivo tenía que expresar su grado de acuerdo o desacuerdo con cada uno de los riesgos presentados

\section{ANÁLISIS Y RESULTADOS}

\subsection{Identificar los niveles de externalización actual y deseado del destino elegido}

Si analizamos los niveles de externalización actual destaca que el nivel de externalización actual es alto en actividades como la animación, la formación, la seguridad y vigi- 
lancia y la lavandería, presentan todas ellas valores medios superiores a 4.5. En cambio, las actividades que presentan un nivel de externalización actual no demasiado alto pero destacable (entre 2 y 4 de media) son: la limpieza de zonas comunes, el departamento de alimentación y bebidas, el mantenimiento y los sistemas de información (véase tabla 1). Aquellas actividades que tienen un nivel de externalización más bajo son aquellas relacionadas con la recepción, la limpieza de habitaciones, la selección del personal y el marketing y ventas con valores menores a 2 en una escala de 1 a 7.

\section{Tabla 1 \\ NIVEL DESEADO DE EXTERNALIZACIÓN ACTUAL Y DESEADO Y DIFERENCIAS DE MEDIAS (T-TEST)}

\begin{tabular}{|l|c|c|l|}
\hline \multicolumn{1}{|c|}{ Actividades } & $\begin{array}{c}\text { Externalización } \\
\text { deseada }\end{array}$ & $\begin{array}{c}\text { Externalización } \\
\text { actual }\end{array}$ & \multicolumn{1}{c|}{$\mathrm{t}$} \\
\hline Recepción & 1.57 & 1.02 & $3.10 * *$ \\
\hline Limpieza de habitaciones & 2.90 & 1.40 & $5.59 * * *$ \\
\hline Limpieza de zonas nobles y comunes & 4.15 & 2.51 & $4.59 * * *$ \\
\hline Lavandería & 5.90 & 5.40 & $2.52 *$ \\
\hline Alimentación y bebidas & 2.49 & 2.27 & 0.95 \\
\hline Mantenimiento (exterior, interior, jardineria) & 3.68 & 2.54 & $4.53 * * *$ \\
\hline Formación & 5.31 & 4.74 & $2.11 *$ \\
\hline Selección del personal y reclutamiento & 2.11 & 1.43 & $3.44 * * *$ \\
\hline Marketing y ventas & 2.90 & 1.46 & $5.51 * * *$ \\
\hline Sistemas de información & 3.92 & 2.77 & $4.90 * * *$ \\
\hline Animación & 5.66 & 5.39 & 1.02 \\
\hline Seguridad y vigilancia & 6.30 & 5.71 & $2.21 *$ \\
\hline
\end{tabular}

*** $\mathrm{p}<0.001, * * \mathrm{p}<0.01 * \mathrm{p}<0.05$.

Los resultados indican que el nivel de externalización deseado es más alto que el nivel de externalización actual para el resto de las actividades. Tienen más potencial de incremento las actividades donde la $t$ de student es mayor, ya que muestran mayores diferencias significativas entre la externalización deseada y la externalización actual; entre ellas se encuentran la limpieza de habitaciones y zonas nobles, el mantenimiento, el marketing y ventas y los sistemas de información.

Estas actividades tienen un mayor potencial de incremento en el futuro. Menos potencial de incremento presentan las actividades que tienen un mayor nivel de externalización actual como son la lavandería, seguridad y vigilancia, animación y la formación. Concretamente en la tabla 2 se analiza la frecuencia del grado de externalización actual de las 749 actividades analizadas en todos los hoteles y se observa que un 47,5\% tiene externalizadas alguna parte y un $52.5 \%$ no tiene externalizada ninguna parte, lo que refleja la importancia que tiene la externalización en la actualidad. 


\section{Tabla 2 \\ GRADO DE EXTERNALIZACIÓN \\ DE LAS ACTIVIDADES ANALIZADAS EN EL ESTUDIO}

\begin{tabular}{|c|c|c|c|}
\hline Grado de externalización & Frecuencia & Porcentaje & Porcentaje acumulado \\
\hline 1 nada externalizado & 393 & 52.5 & $\mathbf{5 2 . 5}$ \\
\hline $\mathbf{2}$ & 45 & 6.0 & $\mathbf{5 8 . 5}$ \\
\hline $\mathbf{3}$ & 41 & 5.5 & $\mathbf{6 4 . 0}$ \\
\hline $\mathbf{4}$ & 43 & 5.7 & $\mathbf{6 9 . 7}$ \\
\hline $\mathbf{5}$ & 35 & 4.7 & $\mathbf{7 4 . 4}$ \\
\hline $\mathbf{6}$ & 26 & 3.5 & $\mathbf{7 7 . 8}$ \\
\hline $\mathbf{7}$ Totalmente externalizado & 166 & 22.2 & $\mathbf{1 0 0 . 0}$ \\
\hline Total actividades & $\mathbf{7 4 9}$ & $\mathbf{1 0 0}$ & \\
\hline
\end{tabular}

1 valores perdidos $=7$

\subsection{Relación entre el grado de externalización con la categoría, tamaño y tipo de hotel}

De forma exploratoria nos interesaba conocer si existía relación entre la externalización con la categoría del hotel, el tipo de hotel (independiente o de cadena) y con el tamaño del hotel. Con respecto a la categoría del hotel realizamos un ANOVA con la intención de comprobar si existen diferencias significativas entre el nivel de externalización con respecto a las categorías del hotel.

Los resultados del ANOVA mostrados en la tabla 3 indican que no existen diferencias significativas entre las diferentes categorías (de 3 a 5 estrellas) en relación a la externalización. Si bien no hay diferencias significativas se observa que cuanto mayor es la categoría mayor es el nivel de externalización. También se contrastó si había diferencias significativas con respecto a la externalización entre los hoteles de cadena y los hoteles independientes. El contraste de medias de la t de student indica que no existen diferencias notables en el grado de externalización de los hoteles de cadena y los hoteles independientes (Véase Tabla 3). En cambio, si analizamos el carácter nacional o internacional de los hoteles de cadena, el contraste de medias muestra que hay diferencias significativas en relación a la externalización con respecto a que la cadena sea nacional o internacional. En concreto, los resultados muestran que los hoteles que pertenecen a una cadena internacional tienen un nivel mayor de externalización que los hoteles de cadena nacional.

Para medir el tamaño del hotel hemos usado el número de habitaciones y dado el carácter cuantitativo de esta variable hemos realizado un análisis de correlaciones entre la externalización media de cada uno de los hoteles y el número de habitaciones. El coeficiente de correlación de Pearson (r) es 0.45 y con un nivel de significación del p $<0.001$, indican que existe una relación positiva entre el tamaño del hotel y el nivel de externalización (Véase Tabla 3). 
Tabla 3

ANÁLISIS ESTADÍSTICOS

\begin{tabular}{|c|c|c|c|}
\hline Variable & Categoría & Media & F \\
\hline \multirow{4}{*}{ Grado de } & 3 & 2.83 & 1.568 \\
externalización & 5 & 3.07 & $\mathrm{p}(0.217)$ \\
\cline { 2 - 4 } & Tipo de hotel & 3.41 & $\mathbf{t}(\mathbf{p})$ \\
\cline { 2 - 4 } & Hoteles de cadena & 3.08 & 1.42 \\
\cline { 2 - 4 } & Hoteles independientes & 2.70 & $\mathrm{p}(0.161)$ \\
\cline { 2 - 4 } & Hoteles de cadena & 2.81 & -2.37 \\
\cline { 2 - 4 } & Hoteles independientes & 3.29 & $\mathrm{p}(0.02)$ \\
\cline { 2 - 4 } & Tamaño & Correlación $(\mathbf{r})$ & $(\mathbf{p})$ \\
\cline { 2 - 4 } & Número de habitaciones & 0.45 & 0.000 \\
\hline
\end{tabular}

\subsection{Beneficios y riesgos de la externalización en los hoteles}

En la tabla 4 se muestran las 12 afirmaciones con sus respectivas medias, medianas y modas obtenidas con el fin de analizar el grado de acuerdo que manifiestan los directivos de los hoteles en relación a los distintos beneficios. Se puede apreciar que son cuatro los beneficios de la externalización que más se perciben entre los directivos, destacando del resto tanto en la media, como en la mediana y en la moda. Estos beneficios son: la externalización ayuda a cubrir necesidades más urgentes del personal (media=4.53), la externalización de servicios favorece la reducción de costes del hotel (media=3.77), la externalización permite incrementar la rentabilidad del hotel (media= 3.65) y la externalización de servicios permite ser más eficiente al hotel (media=3,45). Por el contrario aquéllas menos percibidas y que tienen una puntuación por debajo de 3 , son los beneficios relacionados con: la externalización permite incrementar la calidad de los servicios del hotel (media=2.58), con la externalización nos ayuda a sacar más trabajo adelante y que nuestro personal pueda hacer más cosas (media $=2.79$; media 2.83) y la externalización nos permite liberar de más tiempo para emplearlo en otras tareas (media $=2.84$ ).

Con respecto a los riesgos, planteamos a los encuestados una pregunta en la que incluimos 10 afirmaciones destinadas a conocer la percepción de los directivos sobre los posibles inconvenientes que pueden tener la externalización de actividades. En la tabla 5 se muestran las afirmaciones con sus respectivas medidas de tendencia central (media, mediana y moda), con el fin de analizar el grado de acuerdo que manifiestan los directivos de los hoteles en relación a los distintos riesgos planteados.

Los resultados indican que los principales riesgos estratégicos que perciben los directores de los hoteles son aquellos relacionados en primer lugar con la pérdida de diferenciación de los productos y servicios (media $=4.61$ ), seguidos de que la externalización puede dañar los resultados del hotel (media=3.94). En tercer y cuarto lugar, se perciben como riesgos estratégicos el desconocimiento de la forma de trabajar de los suministradores del servicio $($ media $=3.81)$ y la posible pérdida de control de las actividades (media=3.70). 


\section{Tabla 4 \\ BENEFICIOS DE LA EXTERNALIZACIÓN}

\begin{tabular}{|l|c|c|c|}
\hline \multirow{2}{*}{ Beneficios de la externalización } & \multicolumn{3}{c|}{ Tendencia central } \\
\cline { 2 - 4 } & Media & Mediana & Moda \\
\hline Gracias a la externalización disponemos de más tiempo para realizar las tareas & 2.87 & 3.00 & 1.00 \\
\hline Desempeñamos mejor nuestras tareas gracias a la externalización & 2.82 & 3.00 & 1.00 \\
\hline La externalización de servicios permite ser más eficiente al hotel & $\mathbf{3 . 4 5}$ & 3.00 & 3.00 \\
\hline La externalización ayuda al hotel a cubrir necesidades urgentes de personal & $\mathbf{4 . 5 3}$ & 5.00 & 5.00 \\
\hline Que este hotel externalice facilita que nuestro personal pueda hacer más cosas & 2.83 & 2.00 & 1.00 \\
\hline La externalización ayuda a centrarse en actividades clave del hotel & 3.38 & 3.00 & 1.00 \\
\hline Con la externalización sacamos más trabajo adelante con menos esfuerzo & 2.79 & 3.00 & 3.00 \\
\hline La externalización de servicios favorece la reducción de costes del hotel & $\mathbf{3 . 7 7}$ & 4.00 & 1.00 \\
\hline La externalización aporta cualificación y experiencia al trabajo diario & 3.10 & 3.00 & 3.00 \\
\hline La externalización nos permite liberar tiempo para emplearlo en otras tareas & 2.84 & 3.00 & 3.00 \\
\hline La externalización permite incrementar la rentabilidad del hotel & $\mathbf{3 . 6 5}$ & 3.00 & 2.00 \\
\hline La externalización permite incrementar la calidad de los servicios del hotel & 2.58 & 2.00 & 1.00 \\
\hline
\end{tabular}

\section{Tabla 5}

RIESGOS DE LA EXTERNALIZACIÓN

\begin{tabular}{|l|c|c|c|}
\hline \multicolumn{1}{|c|}{ Riesgos de la externalización } & \multicolumn{3}{c|}{ Tendencia central } \\
\cline { 2 - 4 } & Media & Mediana & Moda \\
\hline $\begin{array}{l}\text { Con la subcontratación se puede perder diferenciación de productos y } \\
\text { servicios }\end{array}$ & $\mathbf{4 . 6 1}$ & 5.00 & 5.00 \\
\hline La subcontratación puede suponer una pérdida en el control de actividades & $\mathbf{3 . 7 0}$ & 3.00 & 5.00 \\
\hline $\begin{array}{l}\text { Desconocemos si la forma de trabajar de las empresas de subcontratación } \\
\text { es la mejor }\end{array}$ & 3.81 & 4.00 & 4.00 \\
\hline $\begin{array}{l}\text { Desconocemos los beneficios que puede suponer una subcontratación de } \\
\text { actividades }\end{array}$ & 2.22 & 2.00 & 1.00 \\
\hline $\begin{array}{l}\text { Es difícil coordinar las actividades subcontratadas con las actividades } \\
\text { realizadas por el hotel }\end{array}$ & 2.87 & 3.00 & 1.00 \\
\hline $\begin{array}{l}\text { Es difícil controlar las operaciones realizadas por las empresas a las que se } \\
\text { subcontrata }\end{array}$ & 2.90 & 2.00 & 1.00 \\
\hline Las decisiones de subcontratar suelen no tener marcha atrás en el hotel & 2.95 & 2.00 & 1.00 \\
\hline $\begin{array}{l}\text { Con las decisiones de subcontratación se pierde autonomía en la toma de } \\
\text { decisiones }\end{array}$ & 2.81 & 2.00 & 1.00 \\
\hline $\begin{array}{l}\text { Las decisiones de subcontratación pueden perjudicar a los recursos y } \\
\text { capacidades del hotel }\end{array}$ & 3.22 & 3.00 & 1.00 \\
\hline La subcontratación puede dañar los resultados del hotel & $\mathbf{3 . 9 4}$ & 4.00 & 1.00 \\
\hline
\end{tabular}


Como menores inconvenientes o riesgos estratégicos percibidos figuran el desconocimiento de los beneficios de la externalización (media=2.22), la pérdida de autonomía que supone externalizar (media=2.81) y el hecho que supone la dificultad en la coordinación y el control de este tipo de decisiones (media=2.87, media $=2.90$ ).

\section{DISCUSIÓN}

En cuanto a los niveles de externalización, si comparamos los resultados del nivel de externalización aplicados en el mismo destino de Gran Canaria realizado hace 13 años por Espino-Rodríguez (2002) se observa un incremento considerable de los niveles de externalización. En nuestro trabajo los resultados indican que casi un $47.5 \%$ de las actividades analizadas tienen alguna parte externalizada mientras que en el 2002, para el mismo destino, sólo había un $26.7 \%$ de las actividades que tenían alguna parte externalizada. En cuanto a la externalización de las actividades, la seguridad y vigilancia y la lavandería eran las actividades que más se externalizaban en el año 2002, según el trabajo de EspinoRodríguez (2002) mientras que en nuestro trabajo se comprueba que las actividades que más se externalizan son la seguridad y vigilancia, y lavandería además de la animación y formación. Actividades estas dos últimas que tenían un bajo nivel de externalización en la pasada década. Por el contrario las actividades que menos se externalizan son aquellas relacionadas con la recepción, la limpieza de habitaciones, la selección del personal y el marketing y ventas. Estas son actividades propias de la gestión hotelera por lo que probablemente exista menos proveedores disponibles en el destino para la atención de las mismas o porque los hoteles hayan decidido invertir en estas actividades para que conformen las competencias básicas del hotel.

Por otra parte, los resultados indican que el nivel deseado de externalización es mayor que el nivel actual en todas las actividades, excepto en la animación y la alimentación y bebidas. Esto indica por un lado que la actividad de animación ya tiene un nivel alto en la externalización actual, mientras que el departamento de alimentación y bebidas es un proceso que está costando que los hoteles den el paso hacia la externalización, bien por desconocimiento o porque no confían en la externalización de esta actividad. En cuanto al resto de actividades destaca el deseo de externalizar las actividades por parte del directivo en caso de encontrar un suministrador adecuado. Estos resultados garantizan que esta estrategia será usada en el futuro e incluso tenderá hacia el incremento.

Los resultados muestran que mientras la categoría no es determinante del grado de externalización sí lo es el tamaño del hotel. Cuanto mayor es el tamaño del hotel mayor es el nivel de externalización. Esto puede ser debido a que los hoteles más grandes pueden aprovechar mejor las economías de escala y adquieren un mayor volumen del servicio, por lo que adquirir servicios externos puede ser más favorable en términos de un mejor precio. Estos resultados coinciden con los obtenidos por Espino-Rodríguez (2004) que demuestra una asociación positiva entre el tamaño del hotel y el nivel de externalización. Asimismo, las cadenas internacionales tienen ligeramente un mayor nivel de externalización que las cadenas nacionales, lo que refleja que el carácter internacional es más propenso a externalizar actividades del hotel debido a la dificultad de unificar y homogeneizar tareas a través de todos los territorios en los que opera. 
En relación con los principales beneficios que perciben los directivos hoteleros encuestados podemos señalar que tienen una percepción más positiva de los beneficios de tipo táctico que de tipo estratégico a diferencia de otros estudios, donde los beneficios estratégicos son más valorados (Espino-Rodríguez y Padrón-Robaina, 2005). Los principales beneficios de la externalización percibidos por los directivos en nuestro trabajo son: (i) la externalización ayuda a cubrir las necesidades más urgentes de personal; (ii) favorece la reducción de costes; (iii) permite incrementar la rentabilidad del hotel; y (iv) permite ser más eficiente. Sin embargo, esta reducción de costes ha ocupado el segundo lugar como beneficio de la externalización a diferencia de otros estudios realizados en el sector, en los que ocupa uno de los lugares más bajos del ranking de los beneficios percibidos de la externalización (Espino-Rodríguez y Padrón-Robaina, 2005; Espino et al., 2012). Esto nos indica que en los hoteles de sol y playa de Gran Canaria, bien por la situación económica de los últimos años, por el aumento de competencia, las características de la demanda, etc., la externalización está obteniendo un carácter cada vez más táctico en consonancia con la búsqueda de eficiencia en sus establecimientos que nos transmitieron los directivos entrevistados para nuestra investigación. Jiang et al. (2006) ya nos proporcionaban una evidencia empírica en su trabajo de que las empresas con actividades externalizadas son más eficientes en sus costes que aquellas que no han externalizado y González et al. (2013) nos señalan que la externalización hace posible el control de costes y se logra una mayor eficiencia.

Esto indica que estamos ante un cambio en la estrategia de externalización de los hoteles, que ha pasado de un enfoque más estratégico en el que prima la calidad, la capacidad de innovación y la capacidad de respuesta a los cambios del mercado, a un enfoque más táctico. Nuestra investigación señala un nuevo marco de la estrategia de externalización frente a otros estudios recientes en otras áreas turísticas (Espino-Rodríguez et al., 2012) cuyos resultados establecen que la externalización permite al hotel complementar sus recursos y capacidades y acceder a un personal más cualificado y experimentado, mientras se benefician de la concentración en sus actividades clave o básicas. La externalización de los hoteles de sol y playa de Gran Canaria se rige por beneficios de naturaleza más táctica que estratégica. Los directivos hoteleros se rigen más por la reducción de costes que por los beneficios de la explotación de sus recursos y capacidades. Estos resultados son similares a los encontrados por Espino-Rodríguez y Padrón Robaina (2005) que demuestran que la externalización está más relacionada con la reducción del coste o razones más de tipo táctico. Aunque otros estudios indican que la externalización está condicionada tanto por razones estratégicas como tácticas (Espino-Rodríguez et al., 2012).

Con respecto a los inconvenientes, los resultados obtenidos avalan los obtenidos en otras investigaciones (Espino-Rodríguez y Padrón-Robaina, 2005; Espino-Rodríguez et al., 2012), siendo los principales riesgos percibidos por los directivos la pérdida de diferenciación de los productos y servicios, el desconocimiento de la forma de trabajar de los suministradores del servicio, la posible pérdida de control de las actividades y el daño que puede suponer la externalización a los resultados del hotel. En cambio comprobamos que los directivos hoteleros no consideran irreversibles las decisiones de externalización si los resultados son insuficientes, incluso estarían dispuestos a recuperar la actividad para desarrollarla internamente de nuevo. 
Este trabajo nos ha permitido identificar la taxonomía de los beneficios y riesgos de la externalización existente en la literatura. Esta taxonomía establece dos beneficios principales de la externalización: la mejora de los recursos y capacidades y la reducción de costes. Con respecto a los riesgos esta taxonomía establece también dos riesgos principales: la pérdida de control sobre el proveedor y la pérdida de ventaja competitiva relacionada con los recursos y capacidades. Por otra parte si analizamos globalmente las ventajas y los riesgos percibidos, observamos que existe una mayor tendencia de los directivos a dar una puntuación superior a los riesgos percibidos que a las ventajas, lo que nos indica que existirá un incremento en la externalización solamente en el caso de que se consigan reducir los riesgos, ya que estos tienen un mayor peso a la hora de tomar las decisiones relacionadas con los límites de la empresa.

\subsection{Implicaciones académicas y prácticas}

Desde un punto de vista académico nuestro estudio amplia el marco teórico sobre los beneficios y riesgos de la externalización en el sector hotelero, construyendo un marco más completo para el estudio de la externalización. Los resultados obtenidos han contrastado un cambio de tendencia en la estrategia de externalización, los motivos para externalizar en la actualidad están siendo más de carácter tácticos que estratégicos. Por su parte, el análisis de la externalización permite identificar nichos de mercado en cuanto a las actividades más demandadas por los hoteles en cuanto a su externalización futura. Desde un punto de vista práctico este trabajo nos permite identificar qué actividades son externalizadas, en cuales de ellas existe un gap entre la externalización deseada y actual y cuales por el contrario son menos externalizadas. Con el análisis de la externalización deseada este trabajo permite identificar nichos de mercado en cuanto a las actividades más demandadas por los hoteles en cuanto a su externalización futura. Esto podría ser usado por proveedores externos que permitan suministrar servicios a los hoteles que demandan un mayor nivel de externalización.

También bajo la óptica de la práctica nos ha permitido identificar las principales ventajas o beneficios que determinan la estrategia de externalización y los principales riesgos que limitan el uso de la misma, lo que proporciona conocimiento y ayuda a los directivos hoteleros en la toma de decisiones sobre la externalización de las actividades de su hotel que también está en función de las dimensiones estratégicas. Aunque nuestra investigación establece que la externalización actual está más relacionada con motivos tácticos que estratégicos, los directivos deben de ser conscientes de que la decisión de externalizar una actividad es una decisión estratégica clave, por lo que deben evitar una perspectiva a corto plazo de ahorro en costes y adoptar un enfoque a largo plazo. El planteamiento estratégico puede proporcionar a los hoteles un mejor resultado que cuando sólo se analizan los costes, pues tiene en cuenta qué tipo de actividades se deben externalizar y cuáles no para conseguir la ventaja competitiva, ya que va a permitir al hotel centrarse en aquellos procesos que realiza mejor que sus competidores o proveedores, es decir, en lo que realmente sabe hacer bien. Nuestro trabajo nos indica un cambio en la tendencia de las razones para la externalización hotelera, que ha pasado de tener un carácter estratégico a táctico. El conocimiento de los profesionales del sector, como son los proveedores de las 
empresas de servicios, de los riesgos percibidos por los directivos se hace imprescindible a la hora de la realización y ejecución del contrato con la finalidad de que se pueda reducir la incertidumbre a la hora de dejar los servicios hoteleros en manos de terceros.

A través de nuestro trabajo los directivos deben entender que si optan por externalizar actividades en sus hoteles conseguirán mayor flexibilidad y dinamismo, por lo que serán capaces de afrontar mejor los cambios y las oportunidades que presenta el sector turístico. Pero también nuestra investigación les muestra que externalizar actividades implica un riesgo para la ventaja competitiva, ya que puede ocurrir que el hotel dependa excesivamente del proveedor para la introducción de nuevos productos o servicios o la posibilidad de que disminuya el potencial de la organización para el aprendizaje organizativo, particularmente en aquellas actividades necesarias para el desarrollo de aquellos negocios y capacidades básicas. Los profesionales del sector deben tener en cuenta que un uso inapropiado de la externalización o el incremento de la misma, puede originar un proceso de declive que puede dejar al hotel sin las capacidades y habilidades necesarias para competir.

\section{BIBLIOGRAFÍA}

ARNOLD, U. (2000): "New dimensions of outsourcing: a combination of transaction cost economics and the core competencies concept", European Journal of Purchasing and Supply Management, vol. 6, n 1, pp. 23-29.

BAILEY, W., MASSON, R. y RAESIDE, R. (2002): “Outsourcing in Edinburgh and the Lothians", European Journal of Purchasing and Supply Management, vol. 8, n 2, pp. 83-95.

BARTHÉLEMY, J. (2001): “The hidden cost of IT Outsourcing”, MIT Sloan Management Review, vol. 42, n ${ }^{\circ}$, pp. 60-69.

BELCOURT, M. (2006): "Outsourcing: the benefits and the risks", Human Resource Management Review, vol.16, n 2, pp. 269-279.

BENGTSSON, L. y DABHOLKAR, M. (2009): "Manufacturing outsourcing and its effect on plant performance - lessons for KIBS outsourcing”, Journal of Evolutionary Economics, vol. 19, $\mathrm{n}^{\circ}$ 2, pp. 231-57.

BETTIS, R., BRADLEY, S. y HAMEL, G. (1992): "Outsourcing and industrial decline”, Academy of Management Executive, vol. 6, pp. 7-22.

BOLAT, T. y YILMAZ, Ö. (2009): “The relationship between outsourcing and organizational performance: is it myth or reality for the hotel sector?", International Journal of Contemporary Hospitality Management, vol. 21, pp. 7-23.

BROOKS, N. (2006): "Understanding IT Outsourcing and its potential effects on IT workers and their environment", The Journal of Computer Information Systems, vol. 46, n ${ }^{\circ} 4, \mathrm{pp} .46-53$.

BUNYARATAVEJ, K., HAHN, E. y DOH, J. (2007): "International offshoring of services: A parity study", Journal of International Management, vol. 13, $\mathrm{n}^{\circ}$ 1, pp 7-21.

BURN, J. y ASH, C. (2000): "Knowledge management strategies for virtual organizations", Information Resources Management Journal, vol.13, $\mathrm{n}^{\circ}$ 1, pp. 15-23. 
BUSTINZA, O., ARIAS-ARANDA, D. y GUTIERREZ, L. (2010): “Outsourcing, competitive capabilities and performance: an empirical study in service firms", International Journal of Production Economics, vol.126, n 2, pp. 276-288.

CAMPOS, B. (2001): El outsourcing de los sistemas y tecnologías de información. Un estudio empírico aplicado a la Comunidad Gallega. Revista Europea de Dirección y Economía de la Empresa, vol.10, n 2, pp. 43-56.

CAREY, P., SUBRAMANIAM, N. y CHING, K.C.W. (2006): "Internal audit outsourcing in Australia", Accounting \& Finance, vol. 46, n' 1, pp. 11-30.

CHASE, R., JACOBS, F. y AQUILANO, N. (2004): Operations Management for Competitive Advantage. 10 th ed., Irwin/McGraw-Hill, Boston, MA.

CONSEJERÍA DE TURISMO DEL GOBIERNO DE CANARIAS (2014): Plazas y establecimientos autorizados según modalidad y categoría.

http://www.gobiernodecanarias.org/presidencia/turismo/estadisticas_y_estudios/

CLARK, T.D., ZMUD, R.W. y MCCRAY, G.E. (1995): The Outsourcing of Information Services: Transforming the nature of business in the information industry. Journal of Information Technology, vol. 10, pp. 221-237.

CRICHTON, E. y EDGAR, D. (1995): Managing complexity for competitive advantage: An IT perspective. International Journal of Contemporary Hospitality Management, vol.7, n ${ }^{\circ}$ 2/3, pp. 12-18.

DAY, G.S. (1994): The capabilities of market-driven organizations. Journal of Marketing, vol. 58, nº 4 , pp. 37-52.

DABHOLKAR, M., BENGTSSON, L., VON HAARTMAN, R. y AHLSTRÖM, P. (2009): "Supplier selection or collaboration? Determining factors of performance improvement when outsourcing manufacturing", Journal of Purchasing and Supply Management, vol.15, $\mathrm{n}^{\circ} 3$, pp. 143-153.

DELMOTTE, J. y SELS, L. (2008): “HR outsourcing: Threat or opportunity?", Personnel Review, vol. 37, $\mathrm{n}^{\circ}$ 5, pp. 543-563.

DESS, G., RASHEED, A., MCLAUGHLIN, K. y PRIEM, R. (1995): “The new corporate architecture", Academy of Management Executive, vol. 9, pp. 7-20.

DOLGUI, A. y PROTH, J.M. (2013): “Outsourcing: definitions and analysis", International Journal of Production Research, vol. 51, n 23-24, pp. 6769-6777.

DOMBERGER, S. (1998): The Contracting Organization: A Strategic Guide to Outsourcing. Oxford University Press, Oxford.

DONADA, C. y NOGATCHEWSKY, G. (2009): "Emotions in outsourcing. An empirical study in the hotel industry", International Journal of Hospitality Management, vol. $28, n^{\circ} 3$, pp. 367-373.

ECERKALE, K. y KOVANCI, A. (2005): "Outsourcing in Human Resources", Journal of Aviation and Space Technologies, vol.2, $\mathrm{n}^{\circ}$ 2, pp. 69-75.

ESPINO-RODRÍGUEZ, T. (2002): Un análisis estratégico de la externalización bajo la visión de la empresa basada en los recursos y capacidades: su aplicación empírica al sector hotelero. Tesis Doctoral. Universidad de las Palmas de Gran Canaria.

ESPINO-RODRÍGUEZ, T. (2004): “The tendency to outsource hotel operations: Strategic reasons and relationship to activity performance and size", Tourism Review, vol. 59, $\mathrm{n}^{\circ} 2$, pp. 17-25. 
ESPINO-RODRÍGUEZ, T. y PADRÓN-ROBAINA, V. (2004): “Outsourcing and its impact on operational objectives and performance: A study of hotels in the Canary Islands", International Journal of Hospitality Management, vol. 23, n 3, pp. 287-306.

ESPINO-RODRÍGUEZ, T. y PADRÓN-ROBAINA, V. (2005): “The management perception of the strategic outsourcing of services: An empirical examination in the hotel sector", The Service Industries Journal, vol. 25, $\mathrm{n}^{\circ}$, pp. 689-708.

ESPINO-RODRÍGUEZ, T. y GIL-PADILLA, A. (2005): "Determinants of information systems outsourcing in hotels from the resource-based view: An empirical study", The International Journal of Tourism Research, vol. 7, $\mathrm{n}^{\circ}$ 7, pp. 35-47.

ESPINO-RODRÍGUEZ, T. y PADRÓN-ROBAINA, V. (2006): “A review of outsourcing from the resource-based view of the firm", International Journal of Management Reviews, vol. 8, $\mathrm{n}^{\circ} 1$, pp. 49-70.

ESPINO-RODRÍGUEZ, T. y GIL-PADILLA, A. (2007): “The impact of outsourcing strategies on information systems capabilities in the hotel industry", The Service Industries Journal, vol. 27, n6, pp.757-777.

ESPINO-RODRÍGUEZ, T., CHUN-LAI, P. y BAUM, T. (2012): "Risks and benefits of outsourcing hotel operations: a comparison between Scotland and Taiwan", Tourism Economics, vol.18, $\mathrm{n}^{\circ} 1$, pp.95-120.

ESPINO-RODRIGUEZ, T. y CHUN-LAI, P. (2014): “Activity outsourcing and competitive strategy in the hotel industry. The moderator role of asset specificity", International Journal of Hospitality Management, vol. 42, pp. 9-19.

FARRELL, D. (2005): “Offshoring: Value creation through economic change”, Journal of Management Studies, vol. 42, pp. 675-683.

FORD, D. y FARMER, D. (1986): "Make or Buy -A key strategic issue”, Long Range Planning, vol. $19, \mathrm{n}^{\circ} 5$, pp. 54-62.

GILLEY, K. y RASHEED, A. (2000): "Making more by doing less: an analysis of outsourcing and its effects on firm performance", Journal of Management, vol. 26, pp. 763-790.

GILLEY, K., GREER, C. y RASHEED, A. (2004): "Human resource outsourcing and organizational performance in manufacturing firms", Journal of Business Research, vol. 57, pp. 232-240.

GONZÁLEZ, R., GASCO, J. y LLOPIS, J. (2005): “Information systems outsourcing risks: A study of large firms", Industrial Management \& Data Systems, vol. 105, $\mathrm{n}^{\circ}$ 1, 45-62.

GONZÁLEZ, R., GASCO, J. y LLOPIS, J. (2011): “What do we know about outsourcing in hotels?", The Service Industries Journal, vol. 31, n 10, pp. 1669-1682.

GONZÁLEZ, R., GASCO, J. y LLOPIS, J. (2013): "Outsourcing and strategy in Spanish town halls: a field study”, Management Decision, vol. 51, nº 1, pp. 97-119.

GROVER, V., CHEON, M.J. y TENG, T.C. (1994): “A descriptive study on the Outsourcing of Information systems functions", Information \& Management, vol. 27, $\mathrm{n}^{\circ}$ 1, pp. 33-44.

GUERRIER, Y., LOCKWOOD, A. (1989): “Core and peripheral employees in hotel operations”, Personnel Review, vol. 18, n 1, pp. 9-15. 
HEMMINGTON, N. y KING, C. (2000): Key dimensions of outsourcing hotel food and beverage services, International Journal of Contemporary Hospitality Management, vol.12, no 4 , pp. 256-261.

HOECHT, A. y TROTT, P. (2006): "Innovation risks of strategic outsourcing”, Technovation, vol. 26, pp. 672-681.

HOLCOMB, T. R. y HITT, M. A. (2007): “Toward a model of strategic outsourcing", Journal of Operations Management, vol. 25, n 2, pp. 464-481.

HOSTELTUR (2015): La externalización en hoteles, tendencia al alza. Hosteltur, 3 de septiembre, 2015.

HOTTMAN, R. y ADAMS, J. (1996): “Go with what you know: outsourcing - reality or myth?”, Bottomline, vol.11, no 7, pp. 22-23.

HU, B. y CAI, L. (2004): "Hotel labor productivity assessment: a data envelopment analysis", Journal of Travel and Tourism Marketing, vol.16, pp. 27-38.

INSTITUTO NACIONAL DE ESTADÍSTICA (2014). Encuesta de ocupación hotelera. http://www.ine.es/jaxi/menu.do?type=pcaxis \&path=\%2Ft11\%2Fe162eoh\&file=inebase

INSTITUTO DE ESTUDIOS TURÍSTICOS (2014). Encuesta de movimientos turísticos en fronteras, FRONTUR

http://www.iet.tourspain.es/WebPartInformes/paginas/rsvisor.aspx?ruta=\%2fFrontur\% $2 \mathrm{fEstructura} \% 2 \mathrm{fAnual} \% 2 \mathrm{fEntradas}+\mathrm{de}+$ turistas $+\mathrm{seg} \% \mathrm{u} 00 \mathrm{fan}+\mathrm{Comunidad}+\mathrm{aut} \% \mathrm{u} 00 \mathrm{f}$ 3noma+de+destino+principal.+-+Ref.204\&par=1\&idioma=es-ES\&anio=2014

JIANG, B., FRAZIER, G. y PRATER, E. (2006): “Outsourcing effects on firms' operational performance: an empirical study", International Journal of Operations and Production Management, vol.26, pp.1280-1300.

JOHNS, N. y LEE-ROSS, D. (1996): "Strategy, risk and decentralization in hospitality operations", International Journal of Contemporary Hospitality Management, vol.8, $\mathrm{n}^{\circ} 2$, pp. 14-16.

KAKABADSE, A. y KAKABADSE, N. (2005): "Outsourcing: current and future trends", Thunderbird International Business Review, vol. 47, n 2, pp.183-204.

KLEPPER R. y JONES W. (1997): Outsourcing information technology, systems and services. Prentice Hall, Upper Saddle River, NJ.

KROES, J. y GHOSH, S. (2010): "Outsourcing congruence with competitive priorities: impact on supply chain and firm performance", Journal of Operations Management, vol. 28, pp.124-143.

LAM, T. y HAN, M. X. (2005): "A study of outsourcing strategy: a case involving the hotel industry in Shanghai, China”, International Journal of Hospitality Management, vol. 24, $\mathrm{n}^{\mathrm{o}} 1$, pp. 41-56.

LAMMINMAKI, D. (2007): "Outsourcing in Australian hotels: a transaction cost economics perspective”, Journal of Hospitality \& Tourism Research, vol. 31, n 1, pp. 73-110.

LAMMINMAKI, D. (2008): “Accounting and the management of outsourcing", Management Accounting Research, vol. 19, n 2, pp. 163-181.

LAMMINMAKI, D. (2009): “An investigation of the role played by frequency and uncertainty in hotel outsourcing decisions", International Journal of Service Technology and Management, vol. 11, n ${ }^{\circ}$, pp. 182-201. 
LAMMINMAKI, D. (2011): “An examination of factors motivating hotel outsourcing”, International Journal of Hospitality Management, vol. 30, pp. 963-973.

LEEMAN, D., REYNOLDS, D. (2012): “Trust and outsourcing. Do perceptions of trust influence the retention of outsourcing providers in the hospitality industry?", International Journal of Hospitality Management, vol. 31, pp. 601-608.

LEI, D. y HITT, M. (1995): "Strategic restructuring and outsourcing: The effect of mergers and acquisitions and LBOs on building firm skills and capabilities", Journal of Management, vol. 21, $\mathrm{n}^{\circ}$ 5, pp. 835-859.

LEWIN, A., MASSINI, S. y PEETERS, C. (2009): "Why are companies offshoring innovation? The emerging global race for talent", Journal of International Business Studies, vol. 40, pp. 901-925.

MAIGA, A. y JACOBS, F. (2004): "The association between benchmarking and organizational performance: an empirical investigation”, Managerial Finance, vol. 30, $\mathrm{n}^{\circ} 8$, pp. 13-33.

MCIVOR, R. (2005): The Outsourcing Process. Strategies for Evaluation and Management. Cambridge University Press, Cambridge.

MCIVOR, R. (2008): "What is the right outsourcing strategy for your process?", European Management Journal, vol. 26, pp. 24-34.

MOL, M., VAN TULDER, R. y BEIJE, P. (2005): “Antecedents and performance consequences of international outsourcing", International Business Review, vol. 14, pp. 599-617.

MOSCHURIS, S. y KONDYLIS, M. (2006): "Outsourcing in public hospitals: a Greek perspective", Journal of Health Organization and Management, vol. 20, $\mathrm{n}^{\circ}$ 1, pp. 4-14.

ORDINI, A. y SILVESTRI, G. (2008): "Recruitment and selection services: efficiency and competitive reasons in the outsourcing of HR practices", International Journal of Human Resource Management, vol. 19, $\mathrm{n}^{\circ}$ 2, pp. 372-391.

OSHRI, I., KOTLARSKY, J. y GERBASI, A. (2011): Can Client Firms Achieve Radical Innovation in IT Outsourcing? ICIS 2011 Proceedings. Paper 4.

ÖZDOĞAN, O. (2006): Outsourcing in terms of Activity Periods in Hotel Enterprises and its Effects on the Financial Performance, PHD Thesis. Dokuz Eylül University, Institute of Social Sciences, Izmir.

PATRONATO DE TURISMO DE GRAN CANARIA (2014): Oferta alojativa de Gran Canaria.http://www.grancanaria.com/patronato_turismo/Listados-de

establecimientos.26171.0.html

PIACHAUD, BS. (2002): "Outsourcing in the pharmaceutical manufacturing process: an examination of the CRO experience”, Technovation, vol. 22, pp. 81-90.

POPPO, L. y ZENGER T. (1998): "Testing alternative theories of the firm: transaction cost, knowledge-based, and measurement explanations for make-or-buy decisions in information services", Strategic Management Journal, vol.19, pp. 853-877.

POWELL, S., TATIKONDA, M. y LIAO, Y. (2006): “A behavioral study of supply manager decision making: factors influencing make versus buy evaluation", Journal of Operations Management, vol. 24, nº 6, pp. 822-838. 
PRAHALAD, C. y HAMEL, G. (1990): “The core competence of the corporation”, Harvard Business Review, vol. 68, pp. 79-93.

PROMSIVAPALLOP, P., JONES, P. y ROPER, A. (2015): "Factors influencing hotel outsourcing decisions in Thailand: modifications to the transaction cost economics approach", Journal of Hospitality \& Tourism Research, vol. 39, n 1, pp. 32-56.

QUÉLIN, B. y DUHAMEL, F. (2003): "Bringing together strategic outsourcing and corporate strategy: outsourcing motives and risks", European Management Journal, vol. $21, \mathrm{n}^{\circ} 5$, pp. 647-661.

QUINN, J. y HILMER, F. (1994): “Strategic outsourcing”, Sloan Management Review, vol. $35, \mathrm{n}^{\circ} 4$, pp. 43-55.

QUINN, J. (1999): "Strategic outsourcing: leveraging knowledge capabilities", Sloan Management Review, vol. 40, $\mathrm{n}^{\circ}$ 4, pp. 9-21.

REDONDO-CANO, A. y CANET-GINER, M.T. (2010): "Outsourcing agrochemical services: economic or strategic logic?" Service Business, vol.4, n 3-4, pp. 237-252.

SANTORO, G. (2015): "Evaluating performance in the hotel industry: An empirical analysis of Piedmont", Journal of Investment and Management, vol. 4, pp. 17-22.

ŞAHIN, A. y BERBEROGLU, N. (2011): "Decision Making Process of Logistic Outsourcing and 3PL Company Selection Criteria, Online Academic", Journal of Information Technology, vol. 2, nº 5, pp. 33-50.

SAUNDERS, C., GEBELT, M. y HU, Q. (1997): “Achieving success in information systems outsourcing", California Management Review, vol. 39, n 2, pp. 63-79.

SCHULER, R. y JACKSON, S. (1987): "Linking competitive strategy with human resource management practices", The Academy of Management Executive vol. 1, pp. 207-219.

SHANI, A., URIELY, N., REICHEL, A. y GINSBURG, L. (2014): "Emotional labor in the hospitality industry: The influence of contextual factors", International Journal of Hospitality Management, vol. 37, pp. 150-158.

TAFTI, M. (2005): Risks factors associated with offshore IT outsourcing. Industrial Management and Data Systems, vol. 105, n 5, pp. 549-560.

TALLMAN, S. y CHACAR, A. (2011): "Communities, alliances, networks and knowledge in multinational firms: a micro-analytic framework", Journal International Management, vol. 17, pp. 201-210.

TARDIVO, G., BRESCIANI, S. y CUGNO, M. (2013): "Sviluppo economico ed infrastrutture: un' analisi empirica delle Province italiane", Sinergie Rivista di Studi e Ricerche, $\mathrm{n}^{\circ}$ 92, pp. 115-137.

TAYLOR, T. (2005): "In defense of outsourcing", Cato Journal, vol. 25, n 2, pp. 367-77.

TETTEH, E. y BURN, J. (2001): "Global strategies for SME-business: Applying the small framework", Logistic Information Management, vol. 14, no 1/2, pp. 171-180.

VENKATESAN, R. (1992): "Strategic Sourcing: To make or not to make", Harvard Business Review, vol. 70, nº 6, pp. 98-107.

VENKATRAMAN, N. (1989): "Strategic Orientation of Business Enterprises: The Construct, Dimensionality, and Measurement", Management Science, vol. 35, $\mathrm{n}^{\circ}$ 8, pp. 942-962. 
WAN, C. S. y SU, A. Y. L. (2010): "Exploring the factors affecting hotel outsourcing in Taiwan", Asia Pacific Journal of Tourism Research, vol. 15, n 1, pp. 95-107.

YILDIZ, S. y HATUN, Z. (2014): "The benefits, risks and effects on performance of the outsourcing: A comparative study of seasonal and permanent hotels", Procedia-Social and Behavioral Sciences, vol. 109, pp. 514-521. 